\title{
Evidence of Hepatitis E Virus Exposure among Seronegative Healthy Residents of an Endemic Area
}

\author{
Mohammad Mushtaq Husain ${ }^{a}$ Ratika Srivastava ${ }^{a}$ Rama Akondy ${ }^{d}$ \\ Rakesh Aggarwal $^{\mathrm{b}}$ Shahid Jameel ${ }^{\mathrm{c}}$ Sita Naik ${ }^{\mathrm{a}}$ \\ Departments of a Immunology and ${ }^{b}$ Gastroenterology, Sanjay Gandhi Postgraduate Institute of Medical Sciences, \\ Lucknow, and 'Virology Group, International Centre for Genetic Engineering and Biotechnology, New Delhi, India; \\ ${ }^{\mathrm{d}}$ Emory Vaccine Center and Department of Microbiology and Immunology, Emory University School of Medicine, \\ Atlanta, Ga., USA
}

\section{Key Words}

Anti-HEV antibodies · ELISPOT assay · Cellular responses

\begin{abstract}
Objective: Hepatitis E virus (HEV) infection is endemic in the Indian subcontinent. Detection of serum anti-HEV IgG has traditionally been used to determine prior exposure to this virus. We studied HEV-specific recall immune responses in healthy subjects with and without detectable anti-HEV IgG. Methods: Memory B and T cells specific for HEV recombinant proteins PORF2 and pORF3 were estimated among healthy subjects residing in an HEV-endemic region using enzyme-linked immunospot (ELISPOT) assays. Results: AntiHEV IgG-negative and anti-HEV IgG-positive healthy subjects had a similar median (range) number of lgG-secreting memory B cells specific for HEV pORF2 [percent of total IgGproducing cells: $0.39(0-13.63)$ vs. $0.83(0-12.78)]$ and HEV pORF3 [0.33 (0.05-12.35) vs. $1.01(0.08-9.48)]$, and of IFN- $\gamma$ secreting memory $T$ cells specific for HEV pORF2 [per one million PBMCs: 16 (0-220) vs. 36.5 (0-474)] and HEV pORF3 [166 (0-957) vs. $70.5(0-533)]$. Eight healthy volunteers residing in the USA and studied as controls lacked detectable T cells specific for HEV pORF2. Conclusion: ELISPOT assays may detect evidence of prior HEV infection in persons resid-
\end{abstract}

ing in areas endemic for this infection and lacking detectable anti-HEV IgG. Seroepidemiological studies that use the serum anti-HEV IgG test may underestimate the frequency of exposure to HEV.

Copyright $\odot 2010$ S. Karger AG, Basel

\section{Introduction}

Infection with hepatitis E virus (HEV) is a common cause of epidemic and sporadic acute hepatitis in several developing countries in Asia and Africa [1,2]. The disease is transmitted by the fecal-oral route, predominantly affects young adults, and is usually self-limiting except in pregnant women in whom severe disease and mortality are common $[1,3]$. The virus contains a 7.2-kb long, single-stranded, positive-sense RNA genome with 3 open reading frames (ORF). Of these, ORF1 codes for viral nonstructural proteins, ORF2 for a 660-amino acid viral capsid protein, and ORF3 for a small phosphoprotein [1].

$\mathrm{HEV}$ infection induces an early IgM response followed closely by appearance of anti-HEV IgG antibodies. The IgM antibodies last for 4-5 months, whereas IgG antibodies persist longer $[4,5]$. Several assays have been developed for the detection of anti-HEV antibodies; how-

\section{KARGER}

Fax +41613061234 E-Mail karger@karger.ch www.karger.com (c) 2010 S. Karger AG, Basel

0300-5526/11/0543-0139\$38.00/0

Accessible online at:

www.karger.com/int
Dr. Sita Naik

Department of Immunology

Sanjay Gandhi Postgraduate Institute of Medical Sciences

Raebareli Road, Lucknow 226014 (India)

Tel. +91 522249 4284, Fax +91 522266 8017, E-Mail sitanaik@ gmail.com 
ever, these have low inter-assay concordance rates [6-8] and appear to have suboptimal sensitivity. In recent years, some better assays have been developed; however, their effectiveness needs further studies $[9,10]$, and there may be need for further improvement.

In regions where hepatitis $\mathrm{E}$ is endemic, anti-HEV IgG seroprevalence rates are only $10-40 \%$, much lower than those expected from frequent occurrences of large outbreaks. This raises the possibility that levels of these antibodies decline with time and gradually become undetectable. In one study, nearly half the sera collected from persons who had been affected during a hepatitis E outbreak 14 years earlier tested negative for anti-HEV IgG [5]. Thus, absence of anti-HEV IgG may not be a reliable marker of lack of prior exposure to HEV, and a better marker may be needed for studying the seroepidemiology of HEV infection. During the course of acute HEV infection, activation of $\mathrm{T}$ cell responses is well documented [11-13]. These responses appear to wane during convalescence along with anti-HEV IgM [14]. In this study, we tried to determine whether enzyme-linked immunospot (ELISPOT) assays for detecting $\mathrm{T}$ cell and $\mathrm{B}$ cell recall (memory) responses to HEV proteins could show prior exposure to $\mathrm{HEV}$ in the absence of detectable anti-HEV IgG.

\section{Methods}

\section{Subjects}

Study subjects included healthy residents of northern India with no history of jaundice in the preceding 5 years, drawn from staff members and healthy blood donors at the Sanjay Gandhi Postgraduate Institute of Medical Sciences, Lucknow, India. Each subject provided blood for separation of serum and peripheral blood mononuclear cells (PBMCs). PBMCs were isolated from heparinized blood by density gradient centrifugation using lymphocyte separation medium (Eurobio, Les Ulis, France). An institutional Ethics Committee approved the study and all subjects provided informed consent.

Anti-HEV IgG and IgM were tested using commercial ELISA kits (MP Diagnostics, Singapore). In brief, test sera were added to wells coated with recombinant HEV antigens. After washing, an anti-human immunoglobulin (anti-IgM or anti-IgG, as appropriate) conjugated with horseradish peroxidase was added. The bound conjugate was detected using a substrate. Cut-offs were calculated as specified by the manufacturer. Healthy persons who tested positive for anti-HEV IgM were excluded.

Enumeration of Total and HEV-Specific IgG-Secreting B Cells For the enumeration of total B cells, PBMCs were stained with fluorescein isothiocyanate (FITC)-conjugated anti-CD19 (BD Biosciences, Pharmingen, San Diego, Calif., USA) and the corresponding isotype control (FACS Calibur; Becton Dickinson,
Singapore) antibodies. For each experiment, 10,000 events were acquired within the PBMC gate and analyzed using the Cell Quest software (Becton Dickinson). For the enumeration of HEV-specific IgG-secreting cells, ELISPOT assay was used. PBMCs (1.5 million) were stimulated with mitogen-containing medium (MCM) that contained polyclonal stimulants [pokeweed mitogen (PWM; 1:1,000; Sigma) and Staphylococcus aureus Cowan strain (SAC; 1:20,000; Sigma) in RPMI containing 10\% FBS] for 3 days.

Triplicate wells of ELISPOT plates (MAIPS4510; Millipore, Billerica, Mass., USA) were coated with coating buffer (PBS; $\mathrm{pH}$ 7.2) alone or anti-IgG Fc antibody (1:100 dilution, AbD Serotec, Germany), pORF2 $(10 \mu \mathrm{g} / \mathrm{ml})$ or pORF3 $(10 \mu \mathrm{g} / \mathrm{ml})$ (pORF2 was expressed in insect cells [15] and pORF3 in Escherichia coli [16], respectively) in coating buffer. The plates were kept at $4^{\circ}$ overnight and then blocked with 10\% CRPMI for $2 \mathrm{~h}$. Stimulated PBMCs in 10\% CRPMI were added to the ELISPOT wells along with a detection antibody [biotin-conjugated goat anti-human $\operatorname{IgG}$ (Sigma)] at 1:100 dilution. Plate was incubated at $37^{\circ}$ in $5 \%$ $\mathrm{CO}_{2}$ for $24 \mathrm{~h}$. Cells were then removed by vigorous washing and spots were developed using streptavidin-HRP (BD Biosciences, N.J., USA) at 1:100 dilution in PBS containing 10\% FBS (Gibco) and 3-amino-9-ethyl carbazole (catalogue No. A-6926; Sigma). ELISPOT plates were scanned using an automated ELISPOT reader (BIOSYS 4000, Germany). Spots obtained in anti-IgG Fc-coated wells represented the total IgG-secreting cells and those in pORF2/3-coated wells represented HEV-specific IgG-secreting cells. Results were expressed as the ratio of antigen-specific (pORF2 or pORF3) IgG-secreting B cells and total IgG-secreting cells, represented as percentage.

ELISPOT Assay for IFN- $\gamma$-Producing Cells

This assay was performed using the human IFN- $\gamma$ ELISPOT set (ELISPOT, catalogue no. 551849; BD ${ }^{\mathrm{TM}}$ ), as per the manufacturer's instructions. In brief, 96-well PVDF plates were coated with purified anti-human IFN- $\gamma$ antibody $(5 \mu \mathrm{g} / \mathrm{ml}$ in sterile PBS). After overnight incubation at $4^{\circ}$, the plates were washed once and blocked with $200 \mu \mathrm{l} \mathrm{CRPMI} \mathrm{containing} 10 \%$ fetal bovine serum (FBS) at $37^{\circ}$ for $2 \mathrm{~h}$. After discarding the blocking solution, $1 \times 10^{5}$ PBMCs were added to each well in a total volume of 200 $\mu \mathrm{l}$ RPMI containing 10\% FBS, 2 mM L-glutamine, 1 mM sodium pyruvate, $25 \mathrm{mM}$ HEPES and antibiotic-antimycotic mixture (Gibco). Stimulants, pORF2 (expressed in insect cells [15]), pORF3 (expressed in E. coli [16]) at $10 \mu \mathrm{g} / \mathrm{ml}$ and phytohemagglutinin (PHA; catalogue No. 10576-015; Gibco) at a 1:100 ratio, were added to triplicate wells; wells without stimulants served as negative control. Plates were incubated at $37^{\circ}$ for $44 \mathrm{~h}$, washed twice with deionized water, and then three times with PBS $+0.05 \%$ Tween-20 (PBST). Biotinylated anti-human IFN- $\gamma$ antibody $(2 \mu \mathrm{g} / \mathrm{ml})$ was then added to each well. After incubation at room temperature for $2 \mathrm{~h}$, the plates were washed with PBST three times and incubated with streptavidin-HRP $(1: 100)$ at room temperature for $1 \mathrm{~h}$. After washing the plates four times with PBST and twice with PBS, 100 $\mu l$ of 3-amino-9-ethyl carbazole (catalogue No. A-6926; Sigma) substrate was added to each well. The reaction was stopped after 10-15 min, the plates were washed thoroughly with distilled water and air dried at room temperature. The number of spot-forming units (sfu) was counted using an automated ELISPOT reader (BIOSYS 4000). In these assays, each spot represented one antigen-specific IFN- $\gamma$-secreting cell. The results were expressed as 
number of sfu per million PBMCs after subtracting the number of spots in unstimulated wells from those in the respective test well.

\section{Studies in US Subjects}

For comparison, 8 healthy volunteers in the US, who had not travelled to an HEV-endemic region, were studied using an ELISPOT assay for HEV pORF2-specific IFN- $\boldsymbol{\gamma}$-producing cells at the Emory Vaccine Center, Atlanta, Ga., USA. These assays differed slightly from those described above in that these used antihuman IFN- $\gamma$ (Clone 1-D1K; Mabtech, Sweden), biotinylated anti-human-IFN- $\gamma$ (Clone 7-B6-1; Mabtech) and avidin-HRP (Vector Labs, Burlingame, Calif., USA). The pORF2 used was identical with that used in studies on Indian subjects. Staphylococcus enterotoxin B (SEB, catalogue No. S4881; Sigma) was used as positive control.

\section{Statistical Analysis}

Data were expressed as median (range). Intergroup comparisons were done using Mann-Whitney U test; $\mathrm{p}<0.05$ was considered significant.

\section{Results}

\section{Total and HEV-Specific IgG-Secreting B Cells}

Thirty-one healthy subjects were studied for B cell responses; 16 subjects were negative for both anti-HEV IgM and IgG [median age 27 (range 23-43) years], whereas 15 subjects were negative for anti-HEV IgM and positive for IgG [27 (23-42) years]. The proportions of total B cells in PBMCs were comparable in the 16 anti-HEV-negative subjects [median $6.90 \%$ (range 2.89-29.36\%)] and the 15 anti-HEV IgG-positive subjects [4.52\% (1.62-28.99\%)]. The frequencies of HEV pORF2- and HEV pORF3-specific IgG-secreting cells were also similar in the two groups (table 1).

\section{HEV-Specific IFN- $\gamma$-Secreting Cells}

Twenty-five healthy subjects were screened for HEVspecific $\mathrm{T}$ cell responses; 13 subjects were negative for both anti-HEV IgM and IgG [27 (19-40) years], whereas 12 subjects were negative for anti-HEV IgM and positive for IgG [28 (25-40) years]. In addition, 8 healthy subjects residing in the US were also studied as controls. Median frequencies of HEV pORF2-specific, IFN- $\gamma$-secreting lymphocytes among PBMCs were similar in subjects with detectable anti-HEV IgG (36.5 [range 0-474] sfu/million PBMCs; $n=12)$ and those without $(16$ [0-220] sfu/million PBMCs; $\mathrm{n}=13$; $\mathrm{p}=0.325$ ) (fig. 1). In comparison, $\mathrm{HEV}$ pORF2-specific IFN- $\gamma$-secreting cells were undetectable in healthy US volunteers (fig. 1), and lower than those in anti-HEV IgG-positive and anti-HEV IgG-negative

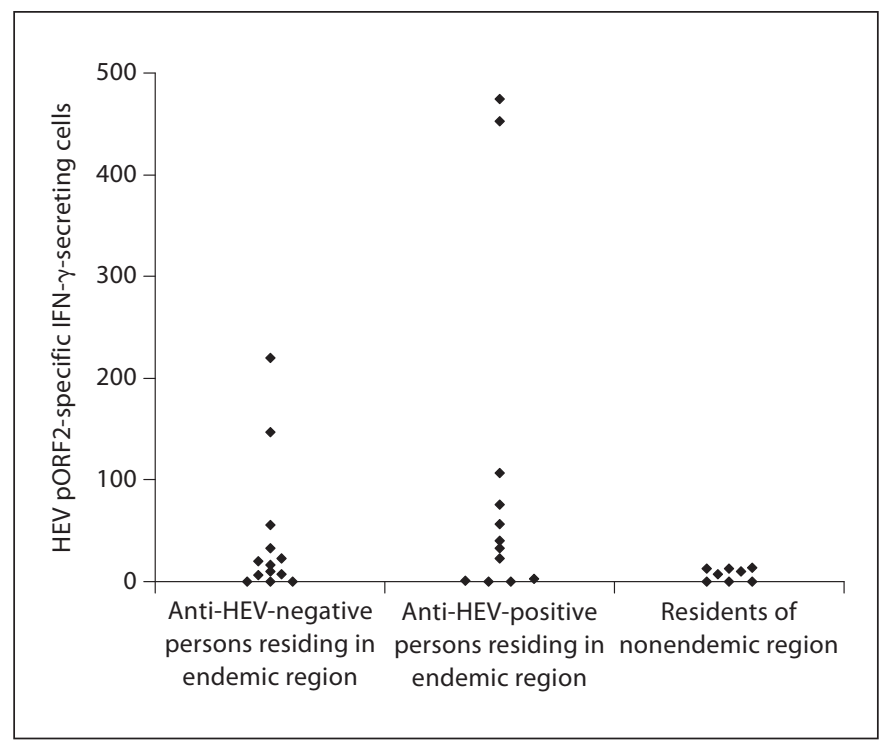

Fig. 1. Number of HEV pORF2-specific, IFN- $\gamma$-secreting cells in healthy Indian subjects with and without anti-HEV antibodies, and healthy subjects residing in the USA, a nonendemic area. Results are expressed as HEV-specific IFN- $\gamma$-secreting cells per million PBMCs. p values using the Mann-Whitney $U$ test for each intergroup comparison are shown below in parentheses: antiHEV IgG-positive vs. anti-HEV IgG-negative Indian subjects $(\mathrm{p}=0.325)$, US subjects vs. anti-HEV IgG-positive Indian subjects $(\mathrm{p}=0.052)$, and US subjects vs. anti-HEV IgG-negative Indian subjects $(\mathrm{p}=0.153)$.

Table 1. Number of anti-HEV IgG-secreting B cells specific for HEV pORF2 and HEV pORF3

\begin{tabular}{llll}
\hline Antigen & \multicolumn{2}{l}{ Serum anti-HEV status } & $\begin{array}{l}\mathrm{p} \\
\text { value }\end{array}$ \\
\cline { 2 - 3 } & $\begin{array}{l}\text { positive } \\
(\mathrm{n}=16)\end{array}$ & $\begin{array}{l}\text { negative } \\
(\mathrm{n}=15)\end{array}$ & \\
\hline HEV pORF2 & $0.83(0-12.78)$ & $0.39(0-3.63)$ & n.s. \\
HEV pORF3 & $1.01(0.08-9.48)$ & $0.33(0.05-12.35)$ & n.s.
\end{tabular}

Data are shown as median (range); results are expressed as HEV-specific spot-forming cells as percent fraction of total IgG spot-forming units.

healthy Indian subjects. However, because of the small number of US controls studied, the difference in number of these cells in this group did not reach statistical significance when compared with those in the anti-HEV IgG-positive and anti-HEV IgG-negative healthy subjects in India ( $p=0.052$ and 0.153 , respectively). 


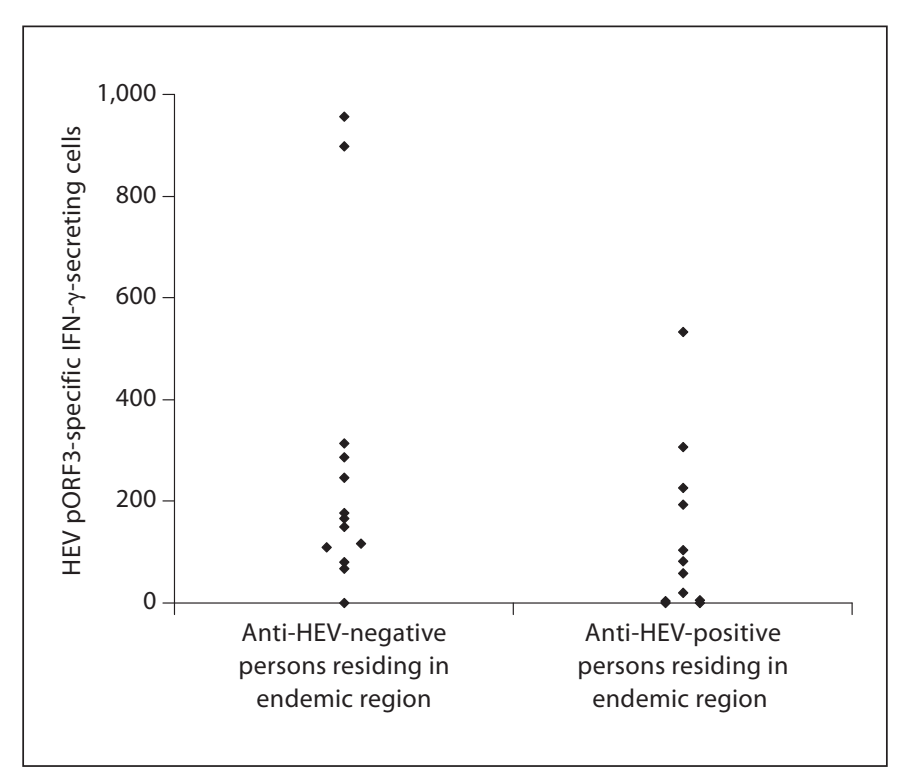

Fig. 2. Number of HEV pORF3-specific, IFN- $\boldsymbol{\gamma}$-secreting cells in healthy Indian subjects with and without anti-HEV antibodies. Results are expressed as HEV-specific IFN- $\gamma$-secreting cells per million PBMCs. Intergroup comparison using the Mann-Whitney $\mathrm{U}$ test, $\mathrm{p}=0.102$.

Median frequencies of HEV pORF3-specific, IFN- $\gamma$ producing cells were also comparable in anti-HEV IgGpositive [70.5 (0-533) sfu/million PBMCs; $\mathrm{n}=12]$ and anti-HEV IgG-negative [166 (0-957) sfu/million PBMCs; $\mathrm{n}=13$ ] subjects (fig. $2 ; \mathrm{p}=0.102$ ).

PBMCs from all the Indian and US volunteers showed good responses to PHA $(5,306[612-8,980]$ sfu/million PBMCs) and SEB (2,088 [690-3,286] sfu/million PBMCs), respectively.

\section{Discussion}

Our data show that a proportion of healthy adults residing in an area where hepatitis $\mathrm{E}$ is endemic and who did not report a history of prior acute hepatitis had demonstrable $\mathrm{HEV}$-specific $\mathrm{B}$ cell and $\mathrm{T}$ cell responses using ELISPOT assays. The magnitude of these responses was similar irrespective of whether the individuals tested positive or negative for anti-HEV IgG.

Detection of pathogen-specific IgG antibodies in serum is often used as a marker of prior exposure of the individual to the particular pathogen, and is widely used for serosurveillance studies. Development of IgG antibody responses is known to be $\mathrm{T}$ cell dependent; this $\mathrm{T}$ cell help is particularly important for affinity maturation of the specific antibody response with time. Exposure to an exogenous agent also leads to generation of long-lived plasma cells and memory cells, which may be responsible for persistence of antibodies for prolonged periods even after elimination of the inciting agent. This has been shown very elegantly among aged survivors of the 1918 H1N1 influenza pandemic [17]. However, the time course of appearance and decline of specific antibodies varies with pathogen. Information on persistence of anti-HEV antibodies is quite limited. As with the detection of specific antibodies, the presence of specific cellular immune responses also reflects prior exposure to a pathogen. Such pathogen-specific cellular immune responses have been used in clinical situations, as attested by the use of tuberculin skin test and assays based on the production of IFN$\gamma$ by immune cells cultured with specific mycobacterial proteins [18].

Our finding of HEV antigen-specific B and T cells using ELISPOT assays in healthy persons who could not recall a history of an illness resembling viral hepatitis indicates that they had been exposed to HEV in the past. This is similar to the detection of anti-HEV antibodies in many persons residing in HEV-endemic areas who do not recall having ever had a hepatitis-like illness, and possibly have had a subclinical infection with HEV. Furthermore, HEVspecific cellular immune responses were detectable among several healthy individuals who lacked anti-HEV antibodies. This finding suggests that these seronegative individuals had also been exposed to HEV in the past, indicating that serological tests for anti-HEV antibodies may be an insensitive marker for the detection of prior exposure to HEV. This could be related either to failure of adequate antibodies to appear following subclinical HEV infection or to their disappearance with the passage of time. The demonstration of detectable B cell memory responses in such patients indicates that the latter is more likely.

Available data on the persistence of anti-HEV antibodies have been few and conflicting. In one study, anti-HEV antibodies were detected in about half of those with a history of HEV infection 14 years ago, but were absent in the other half [5]. In more recent kinetic studies, anti-HEV titers have been shown to decline fairly rapidly [19]. We believe that our findings add to the evidence in favor of the early disappearance of anti-HEV antibody responses. This has implications for the use of seroprevalence studies for understanding the epidemiology of this infection.

We could detect $\mathrm{B}$ cell memory responses in healthy individuals even in the absence of circulating IgG. Our ELISPOT assays for the detection of antibody-producing 
HEV-specific B cells involved polyclonal in vitro stimulation of PBMCs. This would have driven the memory B cell population produced after a previous $\mathrm{HEV}$ infection to differentiate into antibody-producing plasma cells. This ELISPOT assay system would be expected to enumerate all such cells, irrespective of whether these antibodies were produced in vivo or not. This ELISPOT assay may be expected to be more sensitive in assessing prior exposure of a person's immune system to HEV than the detection of anti-HEV IgG ELISA. The absence of detectable HEV-specific IFN- $\gamma$ ELISPOT responses in subjects residing in the USA where HEV infection is less frequent, confirms the specificity of our assays.

Our findings are limited by the small sample size, particularly in the group of persons residing in a nonendemic region. Thus, further larger studies are needed to confirm these results. These studies need to be undertaken both in endemic and nonendemic regions, and in regions where different HEV genotypes are prevalent. It may be particularly relevant to undertake these studies using a common protocol in countries such as Egypt where HEV disease is infrequent though antibody seroprevalence rates are very high, and in India where HEV disease is frequent but seroprevalence rates are relatively lower. Such studies may help in better understanding the cellu- lar and humoral immune responses to HEV, and the epidemiology of HEV infection in these regions.

Though the ELISPOT assays appear to be more sensitive for detection of prior exposure to HEV and hence may be useful for seroepidemiological studies, these assays are more difficult and cumbersome to perform. However, if our findings are confirmed by others, it should be possible to modify the assay by measuring the amount of IFN- $\gamma$ produced instead of enumerating the cells using an ELISPOT.

In conclusion, our data show that ELISPOT assays based on the stimulation of PBMCs with HEV-specific antigens followed by enumeration of cells producing IFN- $\gamma$ or antibodies are more sensitive than serum antibody tests for the detection of prior exposure to this virus. A more widespread use of these assays may help in achieving a better understanding of the epidemiology of this infection.

\section{Acknowledgements}

This work was funded by a research grant from the Department of Biotechnology, Government of India. M.M.H. received a senior research fellowship from the Indian Council of Medical Research, New Delhi.

\section{References}

1 Chandra V, Taneja S, Kalia M, Jameel S: Molecular biology and pathogenesis of hepatitis E virus. J Biosci 2008;33:451-464.

2 Favorov MO, Fields HA, Purdy MA, et al: Serologic identification of hepatitis $\mathrm{E}$ virus infections in epidemic and endemic settings. J Med Virol 1992;36:246-250.

-3 Aggarwal R, Naik S: Epidemiology of hepatitis E: current status. J Gastroenterol Hepatol 2009;24:1484-1493.

-4 Arankalle VA, Chadha MS, Tsarev SA, et al: Seroepidemiology of water-borne hepatitis in India and evidence for a third entericallytransmitted hepatitis agent. Proc Natl Acad Sci USA 1994;91:3428-3432.

5 Khuroo MS, Kamili S, Dar MY, Moecklii R, Jameel S: Hepatitis E and long-term antibody status. Lancet 1993;341:1355.

-6 Mast EE, Alter MJ, Holland PV, Purcell RH: Evaluation of assays for antibody to hepatitis E virus by a serum panel: Hepatitis E Virus Antibody Serum Panel Evaluation Group. Hepatology 1998;27:857-861.

$\checkmark 7$ Myint KS, Endy TP, Gibbons RV, et al: Evaluation of diagnostic assays for hepatitis $\mathrm{E}$ virus in outbreak settings. J Clin Microbiol 2006;44:1581-1583.
-8 Ghabrah TM, Tsarev S, Yarbough PO, Emerson SU, Strickland GT, Purcell RH: Comparison of tests for antibody to hepatitis E virus. J Med Virol 1998;55:134-137.

-9 $\mathrm{Hu}$ WP, Lu Y, Precioso NA, Chen HY, et al: Double-antigen enzyme-linked immunosorbent assay for detection of hepatitis $\mathrm{E}$ virus-specific antibodies in human or swine sera. Clin Vaccine Immunol 2008;15:11511157.

10 Chen HY, Lu Y, Howard T, et al: Comparison of a new immunochromatographic test to enzyme-linked immunosorbent assay for rapid detection of immunoglobulin $\mathrm{m}$ antibodies to hepatitis e virus in human sera. Clin Diagn Lab Immunol 2005;12:593-598.

-11 Naik S, Aggarwal R, Naik SR, et al: Evidence for activation of cellular immune responses in patients with acute hepatitis E. Indian J Gastroenterol 2002;21:149-152.

12 Aggarwal R, Shukla R, Jameel S, et al: T-cel epitope mapping of ORF2 and ORF3 proteins of human hepatitis E virus. J Viral Hepat 2007;14:283-292.

13 Srivastava R, Aggarwal R, Jameel S, et al: Cellular immune responses in acute hepatitis $\mathrm{E}$ virus infection to the viral open reading frame 2 protein. Viral Immunol 2007;20:5665 .
14 Ting Wu, Jun Zhang, Zhi-Jun Su, et al: Specific cellular immune response in hepatitis $\mathrm{E}$ patients. Intervirology 2008;51:322-327.

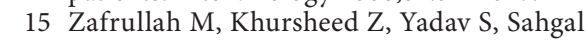
D, Jameel S, Ahmad F: Acidic pH enhances structure and structural stability of the capsid protein of hepatitis E virus. Biochem Biophys Res Commun 2004;313:67-73.

16 Panda SK, Nanda SK, Zafrullah M, Ansari $\mathrm{IH}$, Ozdener MH, Jameel S: An Indian strain of hepatitis E virus (HEV): cloning, sequence, and expression of structural region and antibody responses in sera from individuals from an area of high-level HEV endemicity. J Clin Microbiol 1995;33:2653-2659.

17 Yu X, Tsibane T, McGraw PA, et al: Neutralizing antibodies derived from the $\mathrm{B}$ cells of 1918 influenza pandemic survivors. Nature 2008;455:532-536.

$\rightarrow 18$ Lalvani A: Diagnosing tuberculosis infection in the 21st century: new tools to tackle an old enemy. Chest 2007;131:1898-1906.

19 Myint KS, Endy TP, Shrestha MP, et al: Hepatitis E antibody kinetics in Nepalese patients. Trans R Soc Trop Med Hyg 2006; 100: 938-941. 